\title{
Cacher, dire, montrer, taire - régime de l'imaginaire shakespearien
}

Jean-Marie Maguin

\section{(2) OpenEdition}

Journals

Édition électronique

URL : http://journals.openedition.org/shakespeare/468

DOI : $10.4000 /$ shakespeare.468

ISSN : 2271-6424

Éditeur

Société Française Shakespeare

Édition imprimée

Date de publication : 1 novembre 1983

Pagination : 111-132

Référence électronique

Jean-Marie Maguin, «Cacher, dire, montrer, taire - régime de l'imaginaire shakespearien », Actes des congrès de la Société française Shakespeare [En ligne], 4 | 1983, mis en ligne le 01 janvier 2007, consulté le 04 mai 2019. URL : http://journals.openedition.org/shakespeare/468 ; DOI : 10.4000/ shakespeare.468 
SOCIETE FRANÇAISE SHAKESPEARE

Actes du Congrès 1982

\section{DU TEXTE A LA SCENE : Langages du Théâtre}

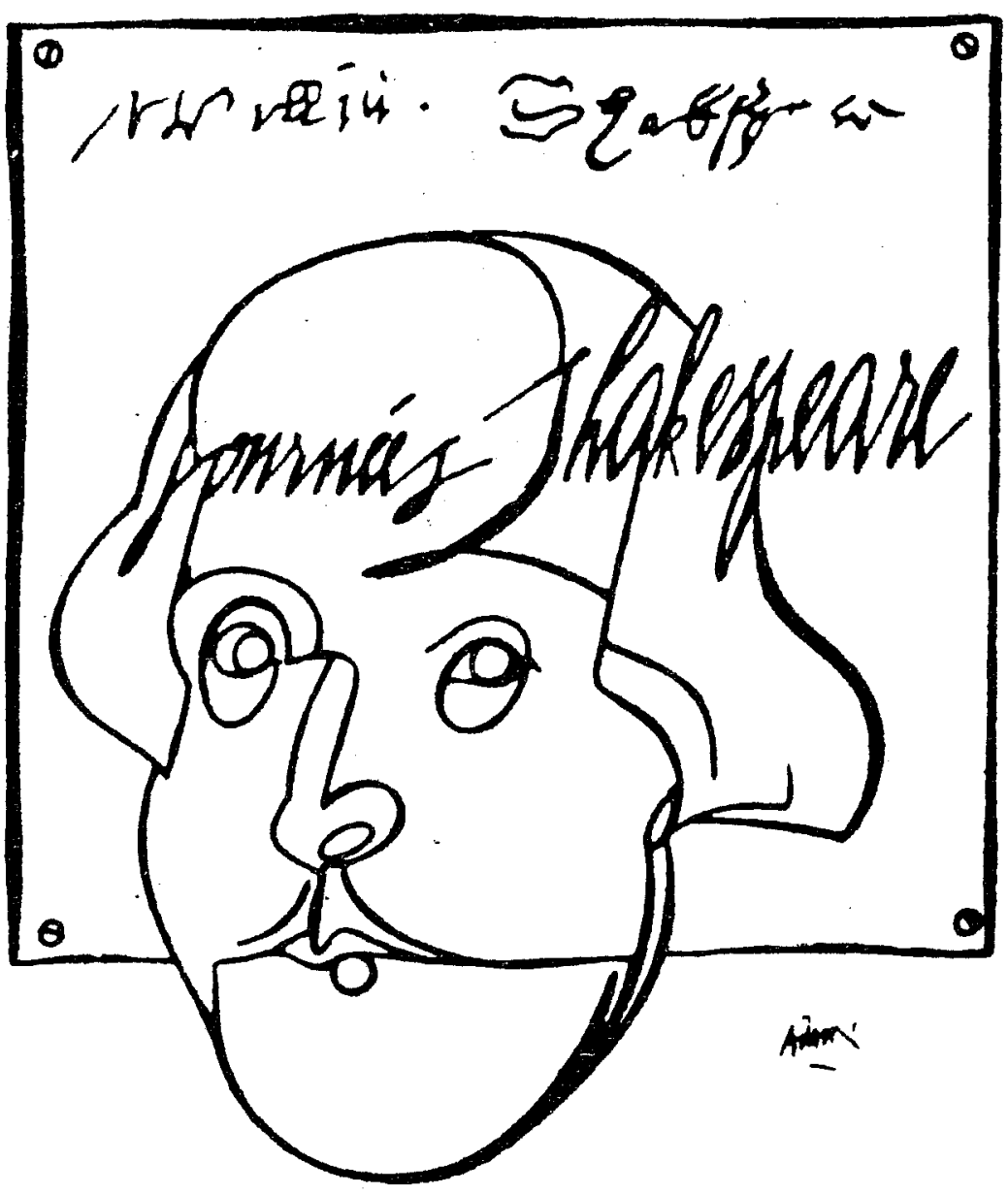

DiRecteur de la publication M.T. Jones - Davies

Publié avec le concours du Centre National de la Recherche Scientifique

JEAN TOUZOT Libraire - Editeur

38 , rue Saint-Sulpice 75278 PARIS CEDEX 061983 


\section{CACHER, DIRE, MONTRER, TAIRE - REGIME DE L'IMAGINAIRE SHAKESPEARIEN *}

La notion d'éphémère est une caractéristique fondamentale de l'opération théâtrale, et peut-être même son surdéterminant ultime. Elle s'impose émotionellement au spectateur dans la mélancolie de l'après-spectacle. Elle parle plus fortement encore à l'intuition métaphysique. Le spectacle fugitif n'est-il pas l'écho de la brièveté de la vie, et ce dans un système étendu d'analogies où tout théâtre est celui de globe, et tout homme histrion appelé à plus ou moins long terme à ne plus être enten du ?

Le dramaturge, perçu en définitive comme un escamoteur, se signale donc par sa capacité de soustraire à tout instant et en dernier ressort tous les signes un moment offerts à nos sens de proximité ${ }^{1}$ : ou ie, vue, et quelquefois aussi odorat, comme alibis de la pseudo-réalité qui occupe l'espace et le temps de la représentation.

Et, comme chacun sait, le «Passez muscade»n'est opérant comme principe de soustraction que si les procédures de présentation ont été d'abord finement organisées, c'est-à-dire sans perdre de vue un seul instant la nécessité du repli, de la résorption. En somme, les espaces et les temps de la manche, de la coulisse et du grenier aux accessoires, parce qu'ils sont des espaces-temps de soustraction et de rétention, comptent autant que ceux de la table ou de la scène de l'illusionniste qui sont les espacestemps d'une présentation fugace.

Je vois dans le mot représentation, appliqué à la performance théâtrale ${ }^{2}$, le témoignage qu'il est impossible de parler du spectacle proposé à notre attention sans avoir l'air de dire que ce spectacle a vaqué, pour toutes ses parties constituantes dans un espace-temps antérieur et de dissolution qui, à lui seul, bâtit le mythe d'une présentation primitive qu'abrite le mot représentation 


\section{Imagination et Imaginaire du Dramaturge}

Elucider les rapports qu'entretient la représentation théâtrale avec la présentation primitive et mythique revient à analyser certaines données du fonctionnement de l'imagination du dramaturge en ce qu'il diffère, par exemple, du fonctionnement de l'imagination du spectateur.

Imaginer, pour le spectateur, revient essentiellement à répondre aux stimuli délibérément - ou accidentellement - proposés à ses sens, à boucher des trous supposés ou réels en faisant appel aux informations stockées dans sa mémoire et provenant aussi bien de son expérience des épisodes antérieurs du spectacle qu'il suit, que de son expérience des spectacles en général et de la vie au sens le plus large. Sa capacité de fantasmer est limitée, sans disparaître totalement, par son choix initial du spectaclefantasme. Elle est, pour l'essentiel, dirigée par le contrat de réceptivité qu'il a passé en prenant sa place.

Imaginer, pour le dramaturge, a une autre portée. Non que son imagination, au contraire de celle du spectateur, soit totalement libre. Elle fonctionne bien évidemment selon des schémas archétypaux et mythiques qui sont autant de données collectives de l'espèce et du milieu culturel, et selon des circuits personnels contraignants qui reflètent les épisodes et les accidents relatifs à l'insertion de l'individu dans son milieu.

La liberté d'imaginer, pour le dramaturge, me paraît d'abord prendre ses racines dans le pari tenu de la genèse, c'est-à-dire dans le refus d'une tentation de ne pas créer. Cette tentation existe, historiquement et par définition, au début de l'élan créateur pour peu qu'il soit saisissable. Elle continue d'exister au fil du travail, et même au-delà de son achèvement comme le montre la toile lacérée et piétinée par le peintre qui la créa, ou des retraits et autodafés d'oeuvres publiées par l'écrivain qui les renie.

Au-delà de cette dichotomie fondamentale du créer ou ne pas créer (c'est-à-dire non pas ne rien faire mais 
retenir un faire virtuel), je voudrais montrer que l'imagination de l'homme de théâtre - et non seulement celle du dramaturge, mais aussi du metteur en scène, de l'acteur, du technicien - continue à tous les stades du travail, et dans son moindre détail, à être confrontée à des choix radicaux du type «oui-non» qui portent sur les modalités de la proclamation du monde imaginaire. Nul ne sera surpris de constater que la pratique du spectacle tend justement à se dissimuler et à nous dissimuler dans ses réponses le caractère dichotomique des options imposées à l'origine.

Pour limiter le débat aux deux sens les plus constamment impliqués dans le spectacle dramatique, la vue et l'ouie, l'homme de théâtre peut montrer ou ne pas montrer, faire entendre ou ne pas faire entendre.Opsis et akoustikon ${ }^{3}$, contiennent donc, chacune en soi, la possibilité d'un refus fondamental. Elles sont en sus, chacune par rapport à l'autre, une alternative. Il est possible de montrer sans rien faire entendre, ou de faire entendre sans rien montrer. Il est aussi possible de montrer ou de faire entendre sans signifier d'emblée, soit que l'on évite d'avoir recours à des termes répertoriés du capital visuel ou acoustique collectif, soit que l'on se situe à un stade de présémiotisation, c'est-à-dire que l'élément optique ou acoustique ne fonctionne pas, ou pas encore, à l'intérieur d'un code établi par la représentation théâtrale donnée. Il est évident, enfin, que l'on peut montrer et faire entendre de façon simultanée et complémentaire comme dans l'expérience vécue ordinairement de la rencontre à fin de communiquer.

Ayant décidé de m'attacher ici à la valeur du négatif, je retiens que mutisme et aveuglement interviennent comme modalisations extrêmes de l'imaginaire dramatique. Les phases de réticence où le projet de communication du monde imaginaire se met radicalement en doute et se renie, ou du moins refuse telle frange possible du spectre optique et acoustique - voire la totalité du spectre de l'une ou l'autre catégorie - me paraissent décisives. 
Ces phases de réticence et de dénégation constituent les champs de cet espace-temps où se dissout entre désir et crainte, amour et haine, ce qui est implicitement, et $a$ posteriori, élevé au rang mythique de présentation primitive.

C'est dire que l'étude de la représentation doit, selon moi, faire quelque place à cet objet hypothétique, et par hypothèse inversé, qu'est la présentation. Tout ce qui est proposé à la vue et à l'ouïe est rejet affirmé d'une tentation de cacher ou de taire, tandis que l'on comprendra peut-être plus naturellement que le non-montré, et le non-dit (ou le non-bruité) résultent d'une censure du montré et du dit (ou du bruité) dans un état antérieur idéal transparaissant dans la représentation.

Une première série dans les exemples qui suivent, et qui sont tous empruntés au théâtre de Shakespeare, va montrer comment opsis et akoustikon jouent l'une par rapport à l'autre un rôle d'alternative, et pour quelles raisons, parfois pratiques. Une deuxième série d'exemples, centrée sur des motifs obsessionnels, montrera comment la prise de relais de l'akoustikon par l'opsis caractérise l'épisode crucial d'histoires d'angoisse qui, de pièce en pièce, se racontent par delà les histoires avouées des intrigues principales et secondaires. Histoires d'angoisses toutes faites du jeu du taire par rapport au dire, du cacher par rapport au montrer. Histoires où cacher revient à dire, et où montrer est taire, tandis que parfois la crise se dénoue dans la coïncidence du dire et du montrer. Je vois apparaître, dans l'enchaînement de ces phases, les données d'un régime - au sens physique du mot de l'imaginaire dramatique, et, ici, de l'imaginaire shakespearien.

Cas simples de variations sur l'Opsis et l'Akoustikon

Le récit d'Enobarbus dans Antony and Cleopatra (II.2.190 et seq. $)^{4}$ est typique du cas de scène à grand 
spectacle - ici, la rencontre entre Antoine et Cléopâtre médiatisée par un narrateur témoin du passé. Le dramaturge évite ainsi la dépense, l'encombrement de la scène et pare à toutes sortes de difficultés d'ordre technologique. Ce faisant, il se retrouve, à l'oralisation près, dans la peau $\mathrm{du}$ chroniqueur, romancier ou poète. Il n'est pas impossible qu'il rêve aux ressources dont disposera plus tard un Cecil B. De Mille.

Dans Julius Caesar (V. 3.9 et seq.), un compromis habile est trouvé, il consiste à ajouter au récit les bruits de la mêlée guerrière avec les sonneries d'attaque et de repli qui ponctuent la fluctuation du sort des armes. Sur ce fond de bruitage, Titinius (12-13) puis Pindarus racontent le combat, qui est censé se dérouler en coulisse, à Cassius dont la vue est trop faible. On sait que Cassius meurt du procédé. En effet, Pindarus a pris pour des ennemis les troupes amies qui enveloppent Titinius parti en reconnaissance. Cassius s'accusant d'avoir causé la mort de son ami se suicide. Le texte de Plutarque ${ }^{5}$ établit seulement que Cassius a mauvaise vue et que, se méprenant sur l'aspect offert par la bataille, il se tue de désespoir. La vue défectueuse de Cassius justifie dans la pièce de Shakespeare l'aveuglement d'une scène militaire à grand spectacle, et bloque le spectateur dans le malentendu en lui retirant à lui aussi le privilège de voir.

Les deux épisodes rapportés qui sont évoqués ici présentent évidemment des circonstances très différentes: faste d'une tactique amoureuse pour le premier, violence guerrière de grande ampleur pour la second. Mais surtout, l'intensité de la médiatisation confiée à Pindarus à un moment-clé du combat provient de la simultanéité du discours et des événements qu'il est censé décrire et que l'accompagnement sonore : instruments, cris, tumulte non identifiable strictement à un camp plutôt qu'à l'autre, suggère de façon pressante en leur assignant comme lieu cet au-delà de l'horizon scénique, ces limbes où le dramaturge manipule à la fois le texte-source de Plutarque, ses personnages, et ses spectateurs. 
A l'inverse de la précision nécessairement apportée dans le détail d'une imagerie verbale de nature compensatoire dès lors que le visuel est amputé on remarque le caractère vague, allusif du verbe quand le visuel est donné en complément. Dans Richard II, Greene et la reine annoncent ainsi l'entrée d'York :

Greene: Here comes the Duke of York.

Queen: With signs of war about his aged neck.

(II. 2. 73-74).

N'importe quel colletin, épaulière, à moins qu'il s'agisse seulement du lourd collier symbole de la régence et arboré en des temps difficiles, tiré du magasin aux accessoires, coulera dans son moule particulier une pâte verbale très plastique, et voulue telle pour éviter toute erreur, complication, ou dépense supplémentaire.

Dans Hamlet, la dernière apparition du fantôme «in his habit as he liv'd» (III.4.137) peut, au gré des mises en scène s'accommoder de n'importe quel costume, jusqu'à la chemise et au bonnet de nuit digne de Scrooge dont l'affubla une année à Stratford un metteur en scène jusqu'au-boutiste. Il est tout à fait vrai que Q1 fournit l'indication «Enter the Ghost in his nightgown》 et cette tenue convient à qui rend une visite nocturne dans les appartements de sa royale épouse.

Macbeth offre un exemple différent de problème relevant de l'opsis. Il s'agit pour l'interprète du rôle principal (puis pour l'actrice incarnant Lady Macbeth dans la scène du somnambulisme) de bien faire voir qu'il voit ce que les autres ne voient pas. C'est la célèbre scène de l'hallucination de la dague, dague dont on peut dire, presque à coup sûr qu'elle n'était visualisée en aucune façon sur la scène élisabéthaine tandis que la technique moderne peut avoir désormais recours à des procédés «sophistiqués» de projection lumineuse. A la moitié exactement des quinze vers présentant l'hallucination (II. 1.33-47), Shakespeare prend soin d'occuper la main de son acteur, naguère refermée sur le vide, par une dague bien visible celle-là et réputée semblable à l'arme immatérielle :«I see 
thee yet, in form as palpable/As this which now I draw» (40-41). Une précaution dans l'ordre de l'opsis est donc prise. Elle soulage à temps acteur et spectateur; l'attention se trouve déplacée de l'objet-fantôme sur l'accessoire observable qui est son double dans le monde de la réalité figurée.

\section{Complexité : les Psychodrames éclatés}

C'est à cette tension variable résultant du recours alternatif ou complémentaire aux catégories de l'opsis et de l'akoustikon, et au degré plus ou moins poussé de concrétisation donné à la proclamation du monde imaginaire mis en scène que je désire m'attacher maintenant, au-delà des exemples élémentaires mais typiques cités jusqu'ici. Comme je l'avais indiqué, les déséquilibres et les équilibres de l'opsis et de l'akoustikon trahissent des mouvements profonds de l'imaginaire personnel du dramaturge, et, d'une pièce à l'autre, une histoire est contée qui diffère de ce que dit chaque pièce en elle-même, et que l'on ne peut espérer voir se dégager clairement de l'enfilade des textes groupés comme pour un volume d'oeuvres complètes.

Les histoires en question sont subliminales parce que constituées de fragments dispersés de représentation. Ce qu'elles signifient concerne évidemment l'imaginaire shakespearien mais agit aussi imperceptiblement sur le nôtre en lui imposant son régime. J'ai choisi de retracer brièvement trois psychodrames éclatés. Le premier pourrait s'intituler «Histoire de l'oreiller»; le second «Les métamorphoses du serpent»; le troisième «Une affaire de poison». La deuxième et la troisième histoires, celle du serpent, et celle du poison, seront traitées simultanément pour être imbriquées de jeu dans la succession de la création dramatique.

Sur les vingt et un emplois du mot pillow dans les pièces, on en relève seulement huit où il intervient dans un contexte de paix ou de repos, paré des connotations 
normalement liées à cet objet de confort. Dans tous les autres cas des déviations plus ou moins subtiles, des perversions manifestes d'usage contaminent l'oreiller, et ce dès le départ de la création dramatique. Dans 2 Henry VI ( $c$. 1590-1592), le cardinal Beaufort à l'article de la mort (III. 2. 373-375) épanche sur son oreiller les secrets d'une âme chargée de crime. Cet oreiller, confident du criminel par défaut de confesseur, se réfère sans doute à l'observation de sommeils agités; peut-être n'est-il qu'une manipulation de l'expression proverbiale to take counsel of one's pillow (1573). Quelque quinze ans plus tard, dans Macbeth - dernière des grandes tragédies - le médecin rattache dans son diagnostique le cas particulier à la loi générale :«infected minds/to their deaf pillows will discharge their secrets» (V.1.69-70). Beaufort se confiait à son oreiller en croyant avouer ses fautes à son souverain. Dans Macbeth plus d'illusion, du moins pour le médecin. L'oreiller est sourd.

C'est dans Richard III (c. 1591-1597) que la traîtrise de l'objet mou et apparemment amical commence d'être révélée. Voici un instantané mélodramatique : les petits princes, dans la Tour, endormis dans les bras l'un de l'autre

Tyrrel: «O thus», quoth Dighton, «lay the gentle babes»;

«Thus, thus», quoth Forrest, «girdling one another

Within their alabaster innocent arms; And in their summer beauty kiss'd each other. A book of prayers on their pillow lay, «Which one», quoth Forest, «almost chang'd my mind $\gg \ldots$ (IV.3.9.-15). Le livre de prières posé sur. l'oreiller, comme il est à l'église ou au château posé sur son coussin, peut-il sauver les innocents ? L'aveu du second meurtrier, aveu non seulement de meurtre mais de méthode nous enlève rapidement tout espoir et nous glace d'horreur : «We smothered/The most replenished sweet work of Nature,/ 
That from the prime creation e'er she fram'd»(17-19). Image de candeur, support de la faiblesse et de l'abandon confiant, support de l'Ecriture et de la prière, l'oreiller est mué en arme pour étouffer les dormeurs.

Dès lors dans l'oeuvre dramatique, il tend à devenir métaphore sinistre et morbide. Dans Titus Andronicus (1594) qui suit vraisemblablement Richard III de quelques mois ou d'un an, si Titus est supposé avoir offert son coeur affectueux pour servir d'oreiller au repos de son petit-fils (V.3.163), les fils de Tamora qui viennent de poignarder Bassianus s'apprêtent à utiliser son corps comme oreiller sur lequel coucher sa femme Lavinia afin de la violer ${ }^{6}$ :

Chiron: Drag hence her husband to some secret hole, And make his dead trunk pillow to our lust

(II.3.129-130).

Dans Lear (1605-1606), l'horrible démon qui persécute Tom par la tentation du suicide cache des couteaux sous son oreiller (III.4.53-54). Dans Macbeth (1606-1607), les oreillers des valets de Duncan servent de reposoir accusateur aux dagues sanglantes que l'épouse de l'assassin y dépose pour détourner les soupçons :

Macbeth : Their hands and faces were all badg'd with blood;

So were their daggers, which, unwip'd, we found Upon their pillows.

(II.3. 102-104).

Glissée sous l'oreiller dans Lear, l'arme fatale émerge et repose sur lui dans Macbeth, après avoir servi.

Dans Cymbeline (c. 1608-1611), on retrouve, euphémisée, la situation évoquée quelque dix-sept ans plus tôt dans Titus Andronicus. A Imogen, déguisée en page, et qui pleure sur le cadavre décapité de Cloten, un capitaine romain demande $: \cdot$ «Who is this/Thou mak'st thy bloody pillow?»(IV.2.362-363). Au-delà, le mot pillow n'est plus jamais utilisé dans l'oeuvre, ni littéralement ni métaphoriquement. Il s'efface sur cette association sanglante et perverse. 
Immédiatement après la naissance de l'obsession de l'oreiller comme arme du crime dans Richard III ou exhaussement morbide d'une violence adultère dans Titus Andronicus, Shakespeare semble vouloir se mettre au clair de son angoisse, hors la scène, dans le poème The Rape of Lucrece (1593-1594). Voici comment Tarquin découvre l'objet de sa passion adultère tandis qu'elle dort d'un sommeil innocent :

Her lily hand her rosy cheek lies under,

Coz'ning the pillow of a lawful kiss;

Who therefore angry, seems to part in sunder,

Swelling on either side to want his bliss :

Between whose hills her head entombed is, Where like a virtuous monument she lies, To be admir'd of lewd unhallowed eyes

(386-392).

Oreiller frustré; oreiller jaloux; oreiller mâle et dressé pour embrasser, pénétrer, envelopper; oreiller-tombe; oreiller de gisant; oreiller nécrophile au bout du compte. Toutes les perversions, toutes les motivations sont rassemblées pour cette animation, pour cette personnification de l'oreiller de Lucrèce qui est l'inverse, en somme, de ces corps humains morts et servant métaphoriquement d'oreiller au tout début de la carrière dramatique dans Titus Andronicus, puis, vers la fin, dans Cymbeline. Ici, dans The Rape of Lucrece, qui par définition ne pose pas les problèmes d'une concrétisation du visuel, tout est dit au fil d'un conceit long, détaillé, et curieux comme un acte d'accusation. Nous savons d'où le danger vient. L'oreiller-arme de Richard III se révèle maniaque sexuel et meurtrier en puissance dans ce dit. Le stade verbal est dépassé, mais modestement, lorsque, dans Titus Andronicus, on entraîne en coulisse le cadavre de Bassianus pour l'usage qu'on sait.

C'est dans Othello (c. 1603-1604) que se fait le passage de l'akoustikon à l'opsis, du mot au geste et à l'accessoire brandi. On se souvient que lago dissuade son maître de se servir du poison pour mettre à mort 
Desdemona. Il conseille au Maure de l'étrangler dans le lit qu'elle est supposée avoir déshonoré. Et Desdemona meurt bien étranglée. Othello lui serre la gorge par charité : «I would not have thee linger in thy pain .../So, so» (V.2. 89-90). Il l'achève. Mais son premier geste fut de l'étouffer (Q1 : he stifles her; F: Smothers her). Dans l'admirable analyse de la pièce que donne Victor Hugo dans son William Shakespeare, il écrit :

Sondez cette chose profonde. Othello est la nuit. Et étant la nuit et voulant tuer, qu'est-ce qu'il prend pour tuer ? Le poison ? la massue, la hache ? le couteau ? Non, l'oreiller. Tuer, c'est endormir. Shakespeare lui-même ne s'est peutetre pas rendu compte de ceci. Le créateur, quelquefois presque à son insu, obéit à son type, tant ce type est une puissance. Et c'est ainsi que Desdemona, épouse de l'homme de Nuit, meurt étouffée par l'oreiller, qui a eu le premier baiser et qui a le dernier souffle (Edition Rencontre, 1968, p. 165).

Davantage que la justice idéale d'abord perçue par Othello (IV.1.205) et le rapport d'harmonie sur lequel insiste Hugo, ce qui me frappe dans le choix de l'oreiller par le jaloux pour accomplir son crime, c'est la continuité obsessionnelle de la symbolique shakespearienne. De ce point de vue - et si l'on cherche à situer le jeu de l'inconscient - on pourrait presque dire que Shakespeare cherche inconsciemment à accorder les circonstances atténuantes à son meurtrier en plaçant sous sa main l'objet où depuis toujours gonflent la jalousie et le désir de tuer. Enfin, c'est le rapport d'ironie qui frappe. L'arme choisie est la preuve formelle - aussi au sens de poétique - de l'innocence de la victime et de sa pureté. L'oreiller ne tue que les purs et les innocents dans le théâtre de Shakespeare Autre ironie tragique, le coussin, ce proche parent de l'oreiller est un emblème reconnu, à la Renaissance, de miséricorde. Falstaff, en s'en coiffant en guise de couronne pour jouer le rôle du monarque, a soin d'emblématiser la 
Justice qui sommeille et ne voit pas le crime ( 1 Henry $I V$, II.4.374). Le mythe personnel que signale la récurrence des associations sinistres apparaît bien en décalage par rapport à la symbolique collective.

Retenons que dans Othello s'exécute sous nos yeux le meurtre dont l'oreiller semble être porteur depuis le début de l'oeuvre dramatique jusqu'aux dernières pièces. Ici, et ici seulement, au moment du passage à l'acte le mot pillow n'est pas prononcé. Ailleurs déjà connu, décrit, connoté, poli dans sa forme littéraire dans The Rape of Lucrece, l'accessoire de thêatre lorsqu'il se fait en scène agent mouvant du drame est l'innommé et l'innommable. Pour l'oreiller, le dire et le montrer ne coincident pas. Au-delà d'Othello et une fois passée la crise, le mot rejoint l'accessoire enfin et par deux fois dans Pericles (16061608). Il retrouve un usage normal d'adjuvant servant de couche à l'orpheline Marina (III.1.67-68), et redressant la tête de Pericles brisé d'émotion lorsqu'il retrouve sa fille (V.1.234). Bien que Cymbeline (c. 1608-1611) voie le retour du cadavre-oreiller, ce dernier ne reçoit - et encore est-ce par erreur - que les pleurs de celle qui se croit veuve et non plus le poids et les assauts du violeur. Si le cauchemar n'a pas disparu, le temps et les ruses de l'imaginaire qui le saisissent tantôt par le mot, et tantôt par le geste dans l'accessoire, ont érodé quelque peu la menace.

«Le plus terrestre des animaux》 d'après la Genèse (III.14-15) mais selon la formulation de Bachelard ( $L a$ terre et les rêveries du repos, Corti, Paris, 1948, p. 262), le serpent, symbolise dans l'oeuvre de Shakespeare les dangers inhérents au sommeil, en complément de l'oreiller dont nous avons pu voir la nocivité. La diversité des vocables employés ne doit pas nous abuser : snake, serpent, adder, ou worm sont employés indifféremment; chacun vise le générique. C'est à terre, allongé pour y reprendre des forces, dans le coma du repos que l'homme est le plus accessible aux entreprises de la bête. Les pouvoirs de cette dernière sont considérables. Macbeth sait que, blessée, elle guérit et redevient dangereuse (III.2.13-15). Dans 
A Midsummer Night's Dream (1594-1598), la reine des fées elle-même ne dédaigne pas de prendre des précautions magiques contre les attaques des serpents (II.2.9-12). Exorcisme d'ailleurs inefficace contre ce prince des serpents qu'est Oberon et qui verse sur les paupières de Titania cette euphémisation du venin qu'est le suc végétal recueilli par Puck et qui engendrera les méprises grotesques. Le serpent est d'ailleurs clairement associé au projet d'Oberon, et la peau de sa mue est décrite comme faisant un vêtement assez ample pour habiller (ou emprisonner?) une fée. Le talus charmant où pousse le thym sauvage et où Titania s'adosse pour son repos nocturne (citation shakespearienne favorite) comporte pourtant cette ombre en appendice :«And there the snake throws her enamell'd skin/Weed enough to wrap a fairy in» (II.1.255-256). L'esprit, qui n'aime pas mélanger symboles fastes et néfastes, préfère oublier les traces de la bête raccrochées au catalogue délicat des fleurs, ou choisit de ne retenir que l'émail multicolore de sa dépouille.

L'association de la fleur et du serpent, qui rêve sans doute de l'association primitive entre le serpent et le fruit défendu à laquelle elle se voudrait même mythiquement antérieure puisque la fleur précède le fruit, est un symbole du mal absolu. Dans cet emblème, la fleur cache le serpent. Lady Macbeth meuble de cette image la rhétorique qu'elle réserve à son époux : «look like th' innocent flower,/But be the serpent under't» (I.5.64-65). Voilà comment l'on peut assassiner Duncan endormi.

Pour en revenir à $A$ Midsummer Night's Dream et à la comédie, Hermia supplie en vain Lysander d'écarter de son sein le serpent qui la menace (II.2.144-155). C'était un mauvais rêve. Plus tard, rencontrant Demetrius, elle l'accuse d'avoir assassiné Lysander pendant son sommeil, et l'image du serpent reparaît de nouveau :

And hast thou kill'd him sleeping ? O brave touch!

Could not a worm, an adder, do so much ?

An adder did it; for with doubler tongue 
Than thine, thou serpent, never adder stung

(III.2.70-73).

Une autre comédie, As You Like It (1598-1600), reprend la menace du serpent en la faisant peser, cette fois, sur un sommeil diurne. C'est par une manière de justice idéale qu'Oliver, qui voulait supprimer son frère en mettant le feu à sa chambre, se trouve à la merci d'un reptile alors qu'il s'est endormi au pied d'un arbre dans la forêt (IV.3.104-110).

Dans Hamlet (1599 - c. 1601), à peu près contemporain de $A s$ You Like It, l'hostilité du serpent envers le sommeil se transforme en agression mortelle :

Ghost : «Tis given out that, sleeping in my orchard,

A serpent stung me - so the whole ear of

Denmark

Is by a forged process of my death

Rankly abus'd - but know, thou noble youth, The serpent that did sting thy father's life

Now wears his crown

$$
\text { (1.5.35 40). }
$$

Le fantôme donne à Hamlet la clé de l'énigme en lui livrant l'identité du serpent. Car le symbole, lui, ne mentait pas. C'était sur le contenu du mot serpent qu'on ne s'entendait pas. Cette oreille de tout le Danemark abusé est la réplique collective de l'oreille royale où fut naguère versé le poison et où bientôt il le sera de nouveau, pour rire, lors de la pièce-dans-la-pièce. Dès lors Claudius est vaincu. Car, lui qui trahit le sommeil du frère par le poison, le voilà obligé de vivre sous l'instance du serpent qui le dénonce et le désigne à la vengeance. Claudius, roi ? Allons donc ! Il n'est, comme dans la fable, que la bête qui a volé une couronne : un serpent couronné. Ajoutons que, comme le serpent, sa seule arme efficace est le poison: celui qu'il met dans la coupe destinée à Hamlet et que Gertrude boit, celui dont est enduit le fer de Laertes. L'épée empoisonnée n'est que l'extension du crochet à venin. On comprend pourquoi Hamlet tient tant à faire vider la coupe par son oncle lors même qu'il lui a passé 
l'épée au travers du corps. Ce n'est pas là une cruauté gratuite, une barbarie de plus destinée aux groundlings. C'est le point d'aboutissement de toute la logique symbolique du danger dans la pièce. Tuer un serpent est une entreprise difficile sauf si, par mégarde et comme pour vous obliger, il vient à se mordre lui-même. Hamlet fait ravaler à Claudius les débordements de son venin. La fatalité qui poussa Claudius à choisir la fable du serpent pour dissimuler le meurtre de son frère endormi ne pouvait mener qu'à cette forme d'auto-destruction forcée.

Examinons bien la pièce dans son ensemble pour y saisir le progrès du venin ou poison. D'abord, fable de la mort du roi, propagée dans ces limbes dramatiques que nous devons bien accepter comme passé absolu de l'histoire racontée au sein de la tragédie. Et, simultanément, élément corrigé de discours dans l'adresse du fantôme à Hamlet à la fin de l'acte I : non pas venin de bête mais poison de meurtrier. Puis accrochage de l'opsis aux variations de l'akoustikon : poison «pour rire»versé dans l'oreille d'un roi de théâtre. Le concret de l'accessoire est atteint mais dans un théâtre au deuxième degré : «they do but jest - poison in jest. No offence i'th' world» (III.2. 229-230). Enfin poison qui gagne la'réalité' du monde de la pièce au bout du fleuret de Laertes pour tuer trois personnages, après avoir envahi la coupe par le biais ironiquement adéquat de l'union émpoisonnée pour éliminer la reine.

Pensons à cette invasion progressive; de la rumeur de l'avant-pièce, au discours de la pièce, à l'accessoire et au discours du théâtre-dans-le-théâtre, au discours et aux accessoires du théâtre tout court. Quelle réaction en chaîne, gagnant les uns après les autres tous les degrés d'une pseudo-réalité ! Seule la convention du théâtre protège, semble-t-il, les spectateurs de la salle des dangers de l'épidémie.

Le poison du mensonge, dont Claudius prétend étourdir le Danemark, est métaphoriquement repris dans Othello. Le maître en est Iago qui empoisonne le sommeil du Maure par ses calomnies contre Desdemona: 
The Moor already changes with my poison :

Dangerous conceits are in their natures poisons, Which at the first are scarce found to distaste, But with a little act upon the blood Burn like the mines of sulphur $[\cdots]$

Look where he comes, not poppy nor

Nor all the drowsy syrups of the world, mandragora, Shall ever medicine thee to that sweet sleep Which thou owedst yesterday

(III.3.330-334 et 335-338)

Le pitoyable aveu d'Othello, dix vers plus loin, «I slept the next night well» (346) dit assez que, déjà, il ne dort plus. N'est-il pas symptomatique de la prudence de Iago de déconseiller au maître, gagné à sa rhétorique, d'utiliser le poison pour punir Desdemona ? En voici un qui ne commettra pas l'erreur de Claudius. Serpent au fond de l'âme, il faut surtout qu'il écarte le poison qui pourrait l'accuser :

Othello: Get me some poison, Iago, this night, I'll not expostulate with her, lest her body and beauty unprovide my mind again, this night, Iago. Iago: Do it not with poison, strangle her in her bed, even the bed she hath contaminated

Othello : Good, good, the justice of it pleases, very

good.

(IV.1.200-205).

Iago a couvert ses traces admirablement. Pour tuer Desdemona, il s'est contenté de mordre un intermédiaire.

Macbeth marque le fond du désespoir. Le héros, dont l'âme est vouée aux scorpions (III.2.36) essaye d'affronter le reptile du danger dynastique en Banquo avec l'insuccès que l'on connaît. Au-delà, la seule tragédie pure - à part Coriolanus - est Antony and Cleopatra. Par l'intermédiaire de Cléopâtre, l'imaginaire shakespearien essaye la ruse là où la force, avec Macbeth, échoua. C'est que le serpent est l'emblème de Cléopâtre et de sa monar- 
chie. Celle qu'Antoine appelle «my serpent of old Nile» (I.5.25) a recours au reptile pour échapper à Octave César. C'est l'alliance avec le serpent pour la première et dernière fois mis en scène. Dans le discours que Cléopâtre adresse à l'aspic toutes les caractéristiques dangereuses s'émoussent ou sont transmuées en pouvoirs bénéfiques :

With thy sharp teeth this knot intrinsicate Of life at once untie : poor venomous fool, Be angry and despatch. O couldst thou speak, That I might hear thee call great Caesar ass, Unpolicied !

(V.2.303-307).

Par une graduelle métamorphose, le serpent est apprivoisé perdant jusqu'à son nom dans la bouche de celle qui se fit une fierté de porter le sobriquet de serpent du Nil. Voici, en retour, humanisée la bête. Cléopâtre, qui cherche le repos, adopte, au sens plein, l'ennemi éternel de l'homme et du repos. A Charmian qui s'exclame lorsqu'elle porte l'aspic à son sein, elle enjoint :

Peace, peace!

Dost thou not see my baby at my breast, That sucks the nurse asleep ?

(V.2.307-309).

Le serpent s'euphémise en nourrisson, la morsure en sucking. Les conditions historiques célèbres du suicide de Cléopâtre qui encoúragent, voire imposent, la seule matérialisation de reptile sur la scène shakespearienne aident le miracle à s'accomplir : le dangereux symbole est complètement subverti ${ }^{7}$.

Après Antony and Cleopatra, le venin fatal reste doux somnifère, non plus par métaphore, mais, cette fois, par substitution. Cymbeline consacre la disparition définitive $\mathrm{du}$ péril sous les auspices du bon docteur, Cornelius, refusant à la reine les drogues mortelles qui lui permettraient d'assouvir ses desseins pour lui remettre d'inoffensifs sédatifs (I.6.33-34). Cornelius réussit d'ailleurs dans Cymbeline le projet misérablement raté par Friar Laurence dans Romeo and Juliet. Imogen se 
réveille en temps utile et l'on découvre que celui qu'elle pleure n'est pas son mari. Serpents ou drogues ne peuvent plus faire de mal à l'homme, au dormeur, ni par le poison du verbe ni par le biais de l'accessoire de théâtre.

$\mathrm{Au}$ terme de cet examen, et pour reprendre la notion de jeu sur les deux grandes catégories, essentielles au théâtre, que sont opsis et akoustikon, constatons que l'imagination du dramaturge puise dans une malle à double ou à triple fond. Il peut en sortir des bruits, de la musique, des mots, ou cela ensemble. Il peut en tirer des objets. Parfois, tout est déballé et ordonné. Il a en permanence la possibilité de ne rien tirer de la boîte. Il a enfin l'obligation d'y faire tout rentrer.

Dès qu'il est extériorisé, le mot, l'objet investi du problème personnel peut être examiné par le dramaturge et par son critique. Il était donc dans la boite puisque le voilà . Une fois disparu, nous savons bien qu'il est quelque part en coulisse, modifié, sans doute, par son dernier avatar public, attendant la réplique, le signe qui lui fournira l'excuse pour reparaître.

Il est évident que mot et objet n'ont pas le même statut. C'est le rapport au corps de l'angoissé qui change lorsque l'on passe de l'un à l'autre, ou que l'on associe les deux. Bien que le critique littéraire doive dans ce domaine laisser la place au psychologue, il peut hasarder que la concrétisation aide à fixer l'angoisse en dehors du corps du sujet. Richard II (IV.1.263) espère se défaire du visage responsable de ses folies passées en brisant le miroir qu'il a fait quérir et où il contemple une image incompréhensiblement sereine, intacte. Bolingbroke fait observer au monarque déchu que ce n'est pas son visage qu'il a brisé mais un reflet de ce visage. Il semblerait donc que le recours à l'accessoire ait une vertu thérapeutique limitée. Le théâtre comme miroir peut lui aussi aider à préciser, grossir, édulcorer, transformer des angoisses le temps d'une représentation, ou de la composition d'une pièce, et pas plus longtemps.

L'exploration de l'imagerie dramatique a trop long- 
temps négligé de s'intéresser à l'opsis de la représentation. Elle doit l'inclure en s'interrogeant constamment sur les rapports entre celle-ci et l'akoustikon dans la mouvance de la performance théâtrale. L'imaginaire du dramaturge a ceci d'original qu'il peut animer de façon éphémère un monde en trois dimensions, et qu'il dispose pour s'exprimer de tous les moyens des arts réunis. Il ne peut être appréhendé que si l'on tient compte de ces circonstances particulières et du fait qu'il agite ses chimères avant et après la représentation, avant et après la composition de telle pièce. On parvient, en rapportant quelques morceaux épars, à saisir d'autres ombres sur une autre scène jouant une pièce qui ne fut jamais écrite en tant que telle. Ce théâtre caché, éclaté, s'il ne peut nous dire qui était le dramaturge, nous apprend tout de même, par bribes et par saccades, ce qu'était le théâtre pour lui et, incidemment, ce qu'est le théâtre en général.

Jean-Marie MAGUIN

NOTES

* Une partie des réseaux d'images analysés ici se trouve đéjà suggérée dans $L$ a nuit dans le théatre de Shakespeare et de ses contemporains, Service de Reproduction des Thèses, Lille, 1980. goût.

1. Pour les différencier, des sens de contact: toucher et

2. On se rapportera à l'analyse très fine de l'impropriété apparente du mot représentation que donne Anne Ubersfeld dans L'école du spectateur, Lire le théatre 2, Editions Sociales, Paris, 1981, p. 10. Anne Ubersfeld écrit :

il ne nous serait pas difficile de poser au départ le théatre comme inversion critique de la notion de représentation: la tâche du théâtre concret étant de construire le modèle réel d'une construction imaginaire : la scène apparaît alors comme un après-coup de l'imaginaire et dans cette perspective le fait de passer ou non par la tex tualité litéraire n'est pas essentiel. 
L'évacuation de la textualité littéraire, même si elle ne va pas sans poser des problèmes, dont traite d'ailleurs Madame Ubersfeld, est une hypothèse intéressante en ce qu'elle permet d'évoquer directement la relation entre l'imaginaire du dramaturge et son projet de représentation. La notion de modèle, dans la fixité et l'unicité qu'elle suggère, me paraît contestable. Je préfère poser par hypothèse que la représentation et l'ensemble idéal des alternatives au niveau du projet contre lesquelles elle a fait ses choix constituent la somme de l'imaginaire créateur de théâtre.

3. Ne trouvant aucun mot consacré par l'analyse pour cette notion importante, je me résigne au barbarisme akoustikon (l'usage français ayant requis le mot «acoustique» à d'autres fins) pour signifier ce qui est audible, livré à l'oreille, et pour faire pendant à l'opsis qui désigne la catégorie du visible. (Pour opsis, voir Patrice Pavis, Dictionnaire du théatre, Termes et concepts de l'analyse the âtrale, Editions Sociales, Paris, 1980, p. 278.)

4. Les références renvoient à la série The Arden Edition of the Works of William Shakespeare (Methuen). Les dates de composition ayant quelque importance pour l'analyse figurent après le titre de la pièce évoquée. Si les pièces sont étudiées, pour l'essentiel, et dans le cadre de chacune des trois séquences d'images envisagées, selon l'ordre de composition généralement admis, c'est la fourchette de dates a quo et ad quem la plus large qui a été retenue pour préserver la marge nécessaire de doute. Ces dates sont empruntées à A. Harbage et S. Schoenbaum, Annals of English Drama, 975-1700, Methuen, London, 1964.

5. Voir Shakespeare's Plutarch, edited by T.J.B. Spencer, Penguin Books, Harmondsworth, 1964 (The Life of Brutus, pp. 159-160).

6. J.C. Maxwell, dans les notes de l'édition «New Arden» (1953) - note 129-130, p. 45, relève après J.D. Ebbs (Modern Language Notes, 66 (1951)) la ressemblance entre le projet de 
Chiron et Demetrius et un passage de The Unfortunate Traveller de Nashe (Works, ed. Mckerrow, II, p. 226) : «Her husbands dead bodie he made a pillow to his abhomination». Par contre la justification de la remarque de J.C. Maxwell selon laquelle «Chiron and Demetrius do not in fact do what they here propose" m'échappe. Cette annotation reflète une tradition qui remonte au moins à la première édition Arden. Ceci pose justement le problème de la prise en compte de l'action en coulisse.

7. Le suicide de Cléopâtre avait, entre autres, frappé Robert Chester qui, dans son Love's Martyr (1601), évoquait

...the snake that Cleopatra used,

The Egyptian queene belov'd of Anthony, That with her breasts deare bloud was nourished ... 